\title{
Inter-relationship of circulating biochemical markers of oxidative stress and comorbid condition in polycystic ovary syndrome.
}

Mahmood Rasool ${ }^{1}$, Rizwan Ahmad², Raazia Rizwan², Arif Malik², Muhammad Asif ${ }^{3}$, Ahmad Zaheer ${ }^{4}$, Abdul Jabbar ${ }^{5}$, Maryam Zain ${ }^{6}$, Muther Mansoor Qaisrani ${ }^{7}$, Tahira Batool Qaisrani ${ }^{7}$, Asim Mehmood $^{8}$, Imran Riaz Malik9, Nawal Helmi ${ }^{10}$, Mustafa Zeyadi ${ }^{10}$, Hani Choudhry ${ }^{10}$, Avi Harlev ${ }^{11}$, Ashok Agarwal $^{11}$, Mohammad Sarwar Jamal ${ }^{12 *}$

${ }^{1}$ Center of Excellence in Genomic Medicine Research, King Abdulaziz University, Jeddah, Saudi Arabia

${ }^{2}$ Institute of Molecular Biology and Biotechnology, The University of Lahore, Lahore, Pakistan

${ }^{3}$ Department of Biotechnology, BUITEMS, Quetta, Pakistan

${ }^{4}$ National Institute for Biotechnology and Genetic Engineering (NIBGE), Faisalabad, Pakistan

${ }^{5}$ Department of Biotechnology, Mirpur University of Science and Technology (MUST), Mirpur, Pakistan

${ }^{6}$ Microbiology and Molecular Genetics Department, the Women University, Multan, Pakistan

${ }^{7}$ Department of Agriculture Science, Ghazi University, Dera Ghazi Khan, Pakistan

${ }^{8}$ Department of Biosciences, COMSATS University Islamabad, Sahiwal Campus, Sahiwal, Pakistan

${ }^{9}$ Departmet of Biotechnology, University of Sargodha, Sargodha, Pakistan

${ }^{10}$ Department of Biochemistry, Cancer Metabolism and Epigenetic Unit, Cancer and Mutagenesis Unit, King Fahd Medical Research Center, King Abdulaziz University, Jeddah, Saudi Arabia

${ }^{11}$ American Center for Reproductive Medicine, Cleveland Clinic, Cleveland, OH, USA

${ }^{12}$ King Fahd Medical Research Center, King Abdulaziz University, Jeddah, Saudi Arabia

\begin{abstract}
Introduction: Polycystic Ovarian Syndrome (PCOS), commonly found in 5\% of all women of reproductive age is a heterogeneous endocrine disorder in which woman's levels of estrogen and progesterone are imbalance leading to the growth of benign masses in the ovaries (ovarian cysts). Rotterdam criteria used to diagnose PCOS include two of three clinical features: 1) oligo- or anovulation, 2) hyperandroginism e.g., hirsutism, acne etc. and 3) sonographic morphologic evidence of polycystic ovaries.

Aim: To investigate the relationship between circulating biomarkers and their heterogeneity in PCOS.

Material and methods: A prospective case control study was conducted including 70 females of age ranging from 20 to $35 \mathrm{y}$ out of which 20 served as healthy controls and 50 were patients of PCOS. Lipid levels, renal profile and antioxidative status of Malondialdehyde (MDA), Catalase (CAT), Superoxide dismutase (SOD) and Reduced glutathione (GSH) was analysed quantitatively. Serum electrolytes $\mathbf{( N a}^{+}$, $\mathrm{K}^{+}, \mathrm{Mg}^{2+}, \mathrm{PO}_{4}{ }^{-3}$ ), anti-oxidants vitamins $\mathrm{C}$ and $\mathrm{E}$ and steroidal hormones like Follicle Stimulating Hormone (FSH), Sex Hormone Binding Globulin (SHBG) were measured by the commercially available kits.

Results: A total of 70 patients were enrolled to the study. Serum electrolytes level like potassium $(\mathrm{K})$ and Phosphorus (P) were significantly increased while antioxidants were decreased in the study group as compared to the control group. Oxidative stress was also increased with higher levels of MDA. Follicle Stimulating Hormone (FSH) and Sex Hormone Binding Globulin (SHBG) were decreased in the study group. The PCOS patients showed statistically significant correlation among the circulating biomarkers like FSH vs. prolactin $\left(\mathrm{r}=0.400^{* *}\right)$.
\end{abstract}




\begin{abstract}
Conclusion: PCOS is found strongly associated with increased lipid peroxidation, oxidative stress, electrolyte imbalance as well as variation in lipid profile in biological system. As the lipid peroxidation increases it increases MDA (end product of lipid peroxidation), significantly it characterize PCOS by quantifying lipid hydroperoxides and therefore it may serve as a good biomarker of oxidative stress of PCOS.
\end{abstract}

Keywords: Polycystic ovarian syndrome (PCOS), Rotterdam criteria, Endocrine, Metabolic disorder, Electrolytes, Oxidative stress, MDA, FSH, SHBG, Prolactin.

Accepted on July 5, 2018

\section{Introduction}

About 5\%-10\% of all the women in their reproductive age suffer from Polycystic Ovarian Syndrome (PCOS) which is a heterogeneous endocrine and metabolic disorder [1-3]. PCOS may involve biological systems other than the reproductive one. It is a well-established cardiovascular disease risk factor as well as a risk factor for various metabolic abnormalities [4-6]. Moreover, pancreatic $\beta$-cell dysfunction and Insulin Resistance (IR) are considered to be major biological aspects that lead to the development of type- 2 diabetes. Hence, women suffering from PCOS were reported to have higher rates for type-2 diabetes in the long term [7-11].

Oxidative stress is considered to be major role player in the pathogenesis of Cardiovascular Disease (CVD) related to PCOS [12-14]. IR has also been documented in patients with PCOS due to oxidative stress [15-17]. Moreover, oxidative stress not only affects the cardiovascular system but also impairs female reproductive physiology, mainly ovulation [18-20]. Hyperglycemia and IR are important factors responsible for the elevated oxidative stress in PCOS patients [21-23]. Lean women with PCOS were also reported to have an elevated oxidative stress without IR [24-26]. The present study was designed to investigate the relationship between circulating biomarkers of stress either enzymatic or nonenzymatic their heterogeneity of PCOS.

\section{Materials and Methods}

Fifty females in the age group of 20-35 y constituted the study group and 20 age-matched females without PCOS constituted the control group. Patients were diagnosed according to the Rotterdam criteria [4]. The experimental protocol was approved by the Research Ethical Committee of The Institute of molecular biology and biotechnology, The University of Lahore. Five milliliter $(5 \mathrm{ml})$ of venous blood sample was taken from each participant. The samples were centrifuged within one hour of collection, after which the sera were separated and stored at $-20^{\circ} \mathrm{C}$ until assayed.

\section{Evaluated parameters}

Lipid and renal profile as well as vitamins $\mathrm{C}$ and $\mathrm{E}$ were estimated by the commercially available kits (Human Diagnostics $\left.{ }^{\circledR}\right)$. Spectrophotometric method was used to measure SOD [27]. Methodology of Ohkawa was employed to measure MDA [28]. Measurement of CAT was performed by Aebi spectrophotometric method [29]. Moron Method been used to measure GSH [30]. Electrolytes $\left(\mathrm{Na}^{+}, \mathrm{K}^{+}, \mathrm{Mg}^{2+}\right.$, $\mathrm{PO}_{4}{ }^{-3}$ ) were estimated by the flame photometer. Hormones like Luteinizing Hormone (LH), FSH, and prolactin were estimated by the commercially available ELISA (Enzyme-Linked Immunesorbent Assay) Kit.

\section{Results}

The study included 70 patients. All the women were from low socioeconomic status as they were at high risk to attain PCOS due to risk for engaging adverse health behaviours, including smoking and poor nutritional diet. Informed written consent was obtained from each patient according to Helsinki declaration. Table 1 represents circulating biochemical markers and various hormones in 2 study groups. Elevated levels of BUN, uric acid as well as BMI in patients suffering from PCOS as compared to controls were observed. Hb level was alleviated in patients $(9.48 \pm 0.21)$ as compared to controls (14.14 $\pm 0.21, \mathrm{p}=0.043)$. All the studied markers were significantly altered between the groups $(\mathrm{P}<0.05)$. Oxidative stress was increased with the increase of MDA and Nitric Oxide (NO) in the study as compared to controls. On the other hand the anti-oxidative stress was decreased with reference to SOD, GSH and CAT as compared to controls.

Elevated levels of total cholesterol (TCh), triglycerides (TG), Low Density Lipoproteins (LDL) and Fasting Blood Sugar (FBS) were observed in PCOS group. Inversely, study group's High Density Lipoprotein (HDL) level (1.39 \pm 0.01$)$ was decreased as compared to controls $(1.73 \pm 0.03, \mathrm{p} \leq 0.05)$. Electrolytes levels were also increased in PCOS patients, i.e., potassium $(\mathrm{K})$ and phosphate $(\mathrm{P})(6.04 \pm 0.13$ and $5.81 \pm 0.08$ respectively, $\mathrm{p} \leq 0.05)$. The levels of calcium $(\mathrm{Ca})$, magnesium $(\mathrm{Mg})$. In the hormonal profile, increased levels of $\mathrm{LH}$, progesterone and prolactin were noticed in the PCOS group compared to controls. Conversely, FSH and Sex Hormone Binding Globulin (SHBG) showed decreased levels in the study group as compared to the control group. The levels of vitamin $\mathrm{E}$ and $\mathrm{C}$ were decreased. As presented in Table 2, statistically significant correlation between FSH and prolactin $\left(\mathrm{r}=0.400^{* *}\right)$ was detected. Similarly strong negative correlation 
Inter-relationship of circulating biochemical markers of oxidative stress and comorbid condition in polycystic ovary syndrome

could be seen in between GSH and progesterone $\left(\mathrm{r}=-0.402^{* *}\right)$, which is a significant finding.

Table 1. Circulating biochemical markers and various hormones in the 2 study groups.

\begin{tabular}{|c|c|c|c|}
\hline Variables & Control $(n=20)$ & $\operatorname{Pcos}(n=50)$ & $P<0.05$ \\
\hline BUN (mg/dL) & $13.75 \pm 0.51$ & $22.44 \pm 0.37$ & 0.022 \\
\hline Uric acid (mg/dL) & $4.01 \pm 0.17$ & $10.17 \pm 0.24$ & 0.041 \\
\hline $\mathrm{Hb}(\mathrm{g} / \mathrm{dL})$ & $14.14 \pm 0.21$ & $9.48 \pm 0.21$ & 0.043 \\
\hline BMI $\left(\mathrm{Kg} / \mathrm{m}^{2}\right)$ & $21.55 \pm 0.35$ & $36.76 \pm 1.09$ & 0.003 \\
\hline MDA (nM/ml) & $1.34 \pm 0.06$ & $3.69 \pm 0.12$ & 0.002 \\
\hline $\mathrm{SOD}(\mathrm{ng} / \mathrm{ml})$ & $0.47 \pm 0.03$ & $0.15 \pm 0.01$ & 0.042 \\
\hline $\mathrm{GSH}(\mathrm{nM} / \mathrm{ml})$ & $9.88 \pm 0.26$ & $2.25 \pm 0.10$ & 0.013 \\
\hline $\mathrm{GPx}(\mu \mathrm{M} / \mathrm{ml})$ & $0.63 \pm 0.07$ & $0.22 \pm 0.01$ & 0.004 \\
\hline CAT ( $\mu \mathrm{M} / \mathrm{mol}$ of protein) & $4.13 \pm 0.18$ & $0.92 \pm 0.08$ & 0.025 \\
\hline $\mathrm{NO}(\mu \mathrm{M} / \mathrm{L})$ & $11.28 \pm 0.30$ & $15.50 \pm 0.23$ & 0.030 \\
\hline TCh (mM/L) & $4.44 \pm 0.08$ & $5.48 \pm 0.13$ & 0.001 \\
\hline $\operatorname{Tg}(\mathrm{mM} / \mathrm{L})$ & $1.24 \pm 0.03$ & $2.59 \pm 0.07$ & 0.022 \\
\hline $\mathrm{LDL}(\mathrm{mM} / \mathrm{L})$ & $2.31 \pm 0.03$ & $2.47 \pm 0.03$ & 0.008 \\
\hline $\mathrm{HDL}(\mathrm{mM} / \mathrm{L})$ & $1.73 \pm 0.03$ & $1.39 \pm 0.01$ & 0.033 \\
\hline Fasting BS (mg/dL) & $90.30 \pm 1.82$ & $130.18 \pm 1.67$ & 0.035 \\
\hline $\mathrm{K}(\mathrm{mmol} / \mathrm{L})$ & $3.88 \pm 0.12$ & $6.04 \pm 0.13$ & 0.001 \\
\hline $\mathrm{Ca}(\mathrm{mmol} / \mathrm{L})$ & $8.81 \pm 0.19$ & $6.01 \pm 0.21$ & 0.025 \\
\hline $\mathrm{Mg}(\mathrm{mmol} / \mathrm{L})$ & $1.77 \pm 0.04$ & $1.41 \pm 0.02$ & 0.039 \\
\hline Phosphate (mmol/L) & $3.87 \pm 0.13$ & $5.81 \pm 0.08$ & 0.032 \\
\hline Vitamin $C(\mu \mathrm{g} / \mathrm{ml})$ & $0.57 \pm 0.01$ & $0.33 \pm 0.01$ & 0.010 \\
\hline Vitamin $E(\mu \mathrm{g} / \mathrm{ml})$ & $0.29 \pm 0.01$ & $0.21 \pm 0.01$ & 0.000 \\
\hline LH (IU/L) & $5.58 \pm 0.12$ & $13.29 \pm 0.49$ & 0.037 \\
\hline FSH (IU/L) & $5.84 \pm 0.09$ & $5.19 \pm 0.15$ & 0.001 \\
\hline Progesterone (ng/dL) & $0.49 \pm 0.01$ & $0.69 \pm 0.00$ & 0.001 \\
\hline SHBG (nmol/L) & $66.48 \pm 0.89$ & $53.59 \pm 1.07$ & 0.020 \\
\hline $\mathrm{PC}(\mu \mathrm{M} / \mathrm{ml})$ & $2.98 \pm 0.11$ & $5.42 \pm 0.15$ & 0.029 \\
\hline Prolidase activity $(\mu \mathrm{M} / \mathrm{L})$ & $60.90 \pm 32.62$ & $69.77 \pm 9.69$ & 0.001 \\
\hline Prolactin (ng/ml) & $15.15 \pm 0.72$ & $21.02 \pm 0.40$ & 0.019 \\
\hline TAS $(\mu \mathrm{mol} / \mathrm{ml})$ & $13.33 \pm 1.27$ & $84.40 \pm 1.40$ & 0.023 \\
\hline
\end{tabular}

BUN: Blood Urea Nitrogen; TCh: Total Cholesterol; Tg: Triglycerides; LDL: Low Density Lipoprotein; HDL: High Density Lipoprotein; MDA: Malondialdehyde; SOD: Superoxide Dismutase; GSH: Reduced Glutathione; CAT: Catalase; GPx: Glutathione Peroxidase; LH: Luteinizing Hormone; FSH: Follicle Stimulating Hormone; SHBG: Sex Hormone Binding Globulin; PC: Protein Carbonyl; TAS: Total Antioxidant Status. Significant $\mathrm{P}<0.05$.

Table 2. Pearson's correlation showing relationship between antioxidants, electrolytes and hormones in PCOS patients.

\begin{tabular}{lll}
\hline Correlation & R value & P value \\
\hline Mg vs. MDA & $-0.324^{*}$ & 0.022 \\
\hline SOD vs. Ca & $-0.450^{* *}$ & 0.001 \\
\hline SOD vs. vitamin C & $0.343^{*}$ & 0.015 \\
\hline SOD vs. PPC & $0.305^{*}$ & 0.031 \\
\hline GSH vs. Ca & $0.326^{*}$ & 0.021 \\
\hline GSH vs. Phos & $-0.315^{*}$ & 0.026 \\
\hline GSH vs. progesterone & $-0.402^{* *}$ & 0.004 \\
\hline CAT vs. Mg & $-0.455^{* *}$ & 0.001 \\
\hline K vs. prolidase & $0.365^{* *}$ & 0.009 \\
\hline Mg vs. Hb & $0.371^{* *}$ & 0.008 \\
\hline NO vs. Tg & $0.363^{* *}$ & 0.010 \\
\hline Vitamin C vs. Tg & $-0.334^{*}$ & 0.018 \\
\hline Vitamin E vs. LDL & $0.339^{*}$ & 0.016 \\
\hline FSH vs. prolactin & $0.400^{* *}$ & 0.004 \\
\hline "Sgnfintp<0.05, Hghly & 0.01. &
\end{tabular}

"Significant $p<0.05,{ }^{* *}$ Highly significant $p<0.01$.

\section{Discussion}

The major findings of the study depict that reduced antioxidants resulted in the increased oxidative stress certainly in the PCOS patients as well as dyslipidemia is a contributing risk factor for future coronary artery diseases and even diabetes. The important components of antioxidative system are GSH, SOD, and CAT which play crucial role into various cellular function. The present study shows the elevated levels of MDA in patients suffering from PCOS as compared to controls and alleviated levels of enzymatic as well as nonenzymatic antioxidants in PCOS. These findings are in complete agreement with Victor's observation [31], who evaluated 20 healthy individuals and 20 women with PCOS for oxidative stress. The authors observed lower levels of GSH. The reduced levels were referred to the association between abnormal ROS production in mitochondria and IR among the PCOS patients. The findings of the current study are also consistent with other studies from Sabuncu, Victor, Kuscu and $\operatorname{Var}[8,12,31]$. Unlike our results, some previous studies did not observe any statistical significant difference in MDA levels in case of women with PCOS as compared to control [32].

LH and FSH levels were considered to be important factors for the diagnosis of PCOS in the form of LH/FSH ratio. Accordingly, in the current study, LH showed elevated levels in PCOS patients while FSH showed decreased levels as compared to control. Although our results correlate with previous studies addressing the $\mathrm{LH} / \mathrm{FSH}$ ratio, this ratio shows low sensitivity for the diagnosis of PCOS $[33,34]$ and therefore it is not used as a diagnosis parameter. However, this study reinforces the use of the LH/FSH ratio as a complementary parameter in PCOS patients. 
Significantly elevated prolactin levels were observed in PCOS patients as compared to the control group. Prolactin is a known role parameter in normal ovulation and elevated levels interfere ovulation. As shown in this study prolactin levels are increased. Previous studies [35] observed no significant prolactin elevation even while were being treated with metformin which is one of the first line treatments of PCOS. Moreover results of current study are in agreement with Filho's reports [36]. Regarding hyperprolactinemia, but according to Filho et al. raised levels of prolactin are not clinical manifestations of PCOS. Significant correlation between FSH and prolactin presents that these are the good biomarkers for PCOS.

\section{Conclusion}

The present study concluded that the women suffering from PCOS have increased lipid peroxidation and oxidative stress as well as they reflected electrolyte imbalance hence increased MDA serves as a significant marker for the identification of disease. Moreover, it emphasizes on the hormonal imbalance so, investigations at hormonal level should be done in the future.

\section{Conflict of Interest}

Declared none

\section{References}

1. Lee H, Oh JY, Sung YA, Chung H, Cho WY. The prevalence and risk factors for glucose intolerance in young Korean women with polycystic ovary syndrome. Endocrine J 2009; 36: 326-332.

2. Sawada M, Masuyama H, Hayata K, Kamada Y, Nakamura K, Hiramatsu Y. Pregnancy complications and glucose intolerance in women with polycystic ovary syndrome. Endocr J 2015; 62: 1017-1023.

3. Ravn P. New paradigms in PCOS: impaired glucose tolerance and cardiovascular risk. Clinical approach. Minerva Ginecol 2015; 67: 217-223.

4. Rotterdam ESHRE/ASRM-Sponsored PCOS consensus workshop group. Consensus on diagnostic criteria and long-term health risks related to polycystic ovary syndrome. Fertil Steril 2004; 81: 19-25.

5. Lazurova I, Figurova J, Lazurova Z. Diagnostics of polycystic ovary syndrome. Vnitr Lek 2015; 61: 40-44.

6. Spritzer PM. New approach polycystic ovary syndrome: reviewing diagnosis and management of metabolic disturbances. Arq Bras Endocrinol Metabol 2014; 58: 182-187.

7. Chang RJ, Nakamura RM, Judd HL, Kaplan SA. Insulin resistance in nonobese patients with polycystic ovarian disease. J Clin Endocrinol Metab 1983; 57: 356-359.

8. Kuayasu NK, Var A. Oxidative stress but not endothelial dysfunction exists in non-obese, young group of patients with polycystic ovary syndrome. Acta Obstet Gynecol Scand 2009; 88: 612-617.
9. Layegh P, Mousavi Z, Farrokh Tehrani D, Parizadeh SM, Khajedaluee $M$. Insulin resistance and endocrinemetabolic abnormalities in polycystic ovarian syndrome: Comparison between obese and non-obese PCOS patients. Int J Reprod Biomed (Yazd) 2016; 14: 263-270.

10. Tokmak A, Kokanali D, Timur H, Kuntay Kokanali M, Yilmaz N.Association between anti-Mullerian hormone and insulin resistance in non-obese adolescent females with polycystic ovary syndrome. Gynecol Endocrinol 2016; 32: 926-930.

11. Wang CC, Chang CJ, Hsu MI. The clinical and biochemical characteristics associated with insulin resistance in non-obese young women. Gynecol Endocrinol 2016; 32: 767-771.

12. Sabuncu T, Vural H, Harma M, Harma M. Oxidative stress in polycystic ovary syndrome and its contribution to the risk of cardiovascular disease. Clin Biochem 2001; 34: 407-413.

13. Kocer D, Bayram F, Diri H. The effects of metformin on endothelial dysfunction, lipid metabolism and oxidative stress in women with polycystic ovary syndrome. Gynecol Endocrinol 2014; 30: 367-371.

14. Bayram F, Kocer D, Ozsan M, Muhtaroglu S. Evaluation of endothelial dysfunction, lipid metabolism in women with polycystic ovary syndrome: relationship of paraoxonase 1 activity, malondialdehyde levels, lowdensity lipoprotein subfractions, and endothelial dysfunction. Gynecol Endocrinol 2012; 28: 497-501.

15. Gonzalez F, Rote NS, Minium J, Kirwan JP. Reactive oxygen species-induced oxidative stress in the development of insulin resistance and hyperandrogenism in polycystic ovary syndrome. J Clin Endocrinol Metab 2006; 91: 336-340.

16. Suresh S, Vijayakumar T. Correlations of insulin resistance and serum testosterone levels with LH: FSH ratio and oxidative stress in women with functional ovarian hyperandrogenism. Indian J Clin Biochem 2015; 30: 345-350.

17. Macut D, Simic T, Lissounov A, Pljesa-Ercegovac M, Bozic I, Djukic T, Bjekic-Macut J, Matic M, Petakov M, Suvakov S, Damjanovic S, Savic-Radojevic A. Insulin resistance in non-obese women with polycystic ovary syndrome: relation to byproducts of oxidative stress. Exp Clin Endocrinol Diabetes 2011; 119: 451-455.

18. Agarwal A, Gupta S, Sharma RK. Role of oxidative stress in female reproduction. Reprod Biol Endocrinol 2005; 3: 28.

19. Al-Gubory KH. Multiple exposures to environmental pollutants and oxidative stress: Is there a sex specific risk of developmental complications for fetuses? Birth Defects Res C Embryo Today 2016; 108: 351-364.

20. Melo AS, Kliemchen J, Junior AA, Ferriani RA, Navarro PA. Oxidative stress and polycystic ovary syndrome: evaluation during ovarian stimulation for ICSI. Reproduction 2016. 


\section{syndrome}

21. Karadeniz M, Erdogan M, Tamsel S, Zengi A, Alper GE, Caglayan O, Saygili F, Yilmaz C. Oxidative stress markers in young patients with polycystic ovary syndrome, the relationship between insulin resistances. Exp Clin Endocrinol Diabetes 2008; 116: 231-235.

22. Celik E, Turkcuoglu I, Ata B, Karaer A, Kirici P, Eraslan S, Taskapan C, Berker B. Metabolic and carbohydrate characteristics of different phenotypes of polycystic ovarysyndrome.. J Turk Ger Gynecol Assoc 2016; 17: 201-208.

23. Victor VM, Rovira-Llopis S, Bañuls C, Diaz-Morales N, Martinez de Marañon A, Rios-Navarro C, Alvarez A, Gomez M, Rocha M, Hernandez-Mijares A. Insulin resistance in PCOS patients enhances oxidative stress and leukocyte adhesion: role of myeloperoxidase. PLoS One 2016; 11: 0151960.

24. Verit FF, Erel O. Oxidative stress in non-obese women with polycystic ovary syndrome: correlations with endocrine and screening parameters. Gynecol Obstet Invest 2008; 65: 233-239.

25. Turan V, Sezer ED, Zeybek B, Sendag F. Infertility and the presence of insulin resistance are associated with increased oxidative stress in young, non-obese Turkish women with polycystic ovary syndrome. J Pediatr Adolesc Gynecol 2015; 28: 119-123.

26. Kurdoglu Z, Ozkol H, Tuluce Y, Koyuncu I. Oxidative status and its relation with insulin resistance in young non-obese women with polycystic ovary syndrome. J Endocrinol Invest 2012; 35: 317-321.

27. Kakkar P, Das B, Viswanathan PN. A modified spectrophotometer assay of superoxide dismutase. Indian J Biochem Biol 1984; 21: 130-132.

28. Ohkawa H, Ohishi N, Yagi K. Assay for lipid peroxides in animal tissues by thiobarbituric acid reaction. J Anal Biochem 1979; 95: 351-358.

29. Aebi H. Catalase in bergmeyer. Methods in enzymatic analysis. Academic Press NY 1984; 276-286.

30. Moron MS, Depierre J, Mannervik B. Levels of glutathione, glutathione reductase and glutathione -S- transferase activities in rat lung and liver. Biochem Biophys Acta 1979; 582: 67-78.

31. Victor VM, Rocha M, Bañuls C, Sanchez-Serrano M, Sola E, Gomez M, Hernandez-Mijares A. DNA damage, DNA susceptibility to oxidation and glutathione level in women with polycystic ovary syndrome. J Clin Endocrinol Metab 2009; 94: 3505-3512.

32. Erdogan M, Karadeniz M, Berdeli A, Alper G, Caglayan O, Yilmaz C. The relationship of the interleukin-6 -174 $\mathrm{G}>\mathrm{C}$ gene polymorphism with oxidative stress markers in Turkish polycystic ovary syndrome patients. J Endocrinol Invest 2008; 31: 624-629.

33. Robinson S, Rodin DA, Deacon A, Wheeler MJ, Clayton $\mathrm{RN}$. Which hormone tests for the diagnosis of polycystic ovary syndrome? $\mathrm{Br}$ J Obstet Gynaecol 1992; 99: 232-238.

34. Cho LW, Jayagopal V, Kilpatrick ES, Holding S, Atkin SL. The $\mathrm{LH} / \mathrm{FSH}$ ratio has little use in diagnosing polycystic ovarian syndrome. Ann Clin Biochem 2006; 43: 217-219.

35. Kazerooni T, Dehghan-Kooshkghazi M. Effects of metformin therapy on hyperandrogenism in women with polycystic ovarian syndrome. Gynecol Endocrinol 2003; 17: 51-56.

36. Filho RB, Domingues L, Naves L, Ferraz E, Alves A, Casulari LA. Polycystic ovary syndrome and hyperprolactinemia are distinct entities. Gynecol Endocrinol 2007; 23: 267-272.

\section{*Correspondence to}

Mohammad Sarwar Jamal

King Fahd Medical Research Center

King Abdulaziz University

Saudi Arabia 